\begin{tabular}{|c|c|c|c|c|c|c|c|c|c|c|}
\hline Nr. & BD. & $\alpha 1900$ & 81900 & $\mathrm{BD}$ & $\begin{array}{l}\text { Größe } \\
\mathrm{PD} \text {. }\end{array}$ & v. Pr. & $\begin{array}{r}\text { Zahl } \\
\text { d. Bb. }\end{array}$ & $\begin{array}{c}\text { Wahrsch. } \\
\text { Fehler }\end{array}$ & $\begin{array}{l}\text { v. } P_{r} . \\
- \text { PD. } \\
\end{array}$ & Bemerlkungen \\
\hline I 89 & $+22: 2531$ & $12^{\mathrm{h}} 53^{\mathrm{m}} 49^{\mathrm{s}}$ & $+22^{\circ} 35^{\prime}: 2$ & $6 \mathrm{~m} .8$ & $7 \div 48$ & $7 \cdot 37$ & 4 & 0.039 & $-.0^{\mathrm{m}} \mathrm{I} \mathrm{I}$ & \\
\hline 190 & +28.2170 & $1253 \quad 56$ & $+28 \quad 0.5$ & $7 \cdot 5$ & 7.94 & 7.88 & 5 & 0.034 & -0.06 & \\
\hline 191 & $+20.278 \mathrm{I}$ & 125359 & $+20 \quad 6.3$ & 9.0 & - & 8.28 & 4 & 0.013 & - & \\
\hline 192 & +28.2171 & $12 \quad 54 \quad 45$ & +2837.6 & $7 \cdot 3$ & 7.29 & $7 \cdot 3^{2}$ & 5 & 0.036 & +0.03 & \\
\hline I 93 & +29.2350 & $12 \quad 54 \quad 47$ & +2846.6 & 8.1 & - & 7.96 & 6 & 0.035 & - & \\
\hline I 94 & +22.2532 & $12 \quad 5523$ & +226.6 & $7 \cdot 3$ & $7 \cdot 72$ & $7.6 \mathrm{I}$ & 4 & 0.022 & -0.11 & \\
\hline 195 & +31.2434 & I $55 \quad 3^{2}$ & +3119.2 & 5.2 & 5.09 & 4.88 & 4 & 0.041 & $-0.2 \mathrm{I}$ & \\
\hline 196 & +24.2522 & I2 $55 \quad 45$ & +2411.8 & $7 \cdot 5$ & 8.42 & 8.17 & 4 & 0.019 & -0.25 & \\
\hline I 97 & +19.2622 & 125546 & +1855.1 & 6.5 & 6.31 & 6.46 & 4 & 0.023 & +0.15 & \\
\hline 198 & $+25.25^{8} 3$ & I $2 \quad 56$ 1 3 & $+2451 \cdot 3$ & 7.4 & 8.00 & 7.72 & 4 & 0.015 & -0.28 & \\
\hline I 99 & +22.2537 & I $2 \quad 56 \quad 43$ & +2 I 48.8 & 7.0 & 7.12 & 7.10 & 4 & 0.016 & -0.02 & \\
\hline 200 & +29.2357 & $12 \quad 57 \quad 19$ & +2932.0 & 8.0 & - & 8.69 & 4 & 0.025 & - & \\
\hline 201 & +24.2528 & I 25740 & +24 & 6.8 & 7.82 & $7 \cdot 59$ & 4 & $0.04 \mathrm{I}$ & -0.23 & \\
\hline 202 & +23.2528 & $1257 \quad 4^{6}$ & +2330.1 & $7 \cdot 5$ & 8.22 & 8.05 & 4 & 0.045 & -0.17 & \\
\hline 203 & +23.2530 & I $258 \quad 7$ & +2310.5 & 6.7 & 7.06 & 7.09 & 4 & 0.019 & +0.03 & \\
\hline 204 & +23.2531 & $1258 \quad 11$ & +2344.3 & 7.0 & $7 \cdot 55$ & $7 \cdot 47$ & 4 & 0.040 & -0.08 & \\
\hline 205 & +24.2531 & I $2 \quad 5^{8} \quad 22$ & $+24 \quad 2$ I. 3 & 6.5 & 7.08 & 7.04 & 4 & 0.043 & -0.04 & \\
\hline
\end{tabular}

Berlin, 1902 Nov. 14.

F. von Prittuitz.

Sternschnuppenbeobachtungen in Port Adelaide.

Von H. Hardh.

Auf einer Weltumsegelung, die ich in den Jahren 1899 bis 1900 vorgenommen hatte, kam ich dazu einige Wochen in der Stadt Port Adelaide in Süd-Australien zuzubringen. Einen Teil dieser Zeit habe ich zum Beobachten der Sternschnuppen benutzt. Die scheinbaren Bahnen gegen die Sterne wurden dabei auf einer Karte in gnomonischer Projektion nach R. A. Proctor eingezeichnet.

Die Resultate dieser Beobachtungen, über welche das
Nähere in „Öfversigt af Finska Vet.-Soc. Förhandlingar, Bd. XLIII \&u finden ist, werden hier in der Kürze mitgeteilt.

Die folgende Tafel enthält nebst den verbesserten Positionen der aus den Beobachtungen bestimmten Radiationspunkte die Korrektionen für die Zenitattraktion und die tägliche Aberration, die Anzahl der zur Bestimmung dienenden Sternschnuppen, sowie die Epochen:

\begin{tabular}{|c|c|c|c|c|c|c|c|c|}
\hline \multirow{3}{*}{$\begin{array}{c}\text { Ra- } \\
\text { diant } \\
\text { Nr. }\end{array}$} & \multirow{3}{*}{$\begin{array}{c}\text { Epoche } \\
\text { I9o0 }\end{array}$} & \multirow{3}{*}{$\begin{array}{c}\text { Anzahl } \\
\text { der Stern- } \\
\text { schnuppen }\end{array}$} & \multicolumn{2}{|c|}{ Position 1900.0} & \multicolumn{4}{|c|}{ Korrebtionen } \\
\hline & & & \multirow{2}{*}{$\alpha$} & \multirow{2}{*}{8} & \multicolumn{2}{|c|}{ für die Zenitattraktion } & \multicolumn{2}{|c|}{ fiir die tägl. Aberration } \\
\hline & & & & & $\Delta \alpha$ & $\Delta 8$ & $\Delta^{\prime} \alpha$ & $\Delta^{\prime} \delta$ \\
\hline $\mathbf{I}$ & Jan. 25 & 8 & $100 \% 2$ & $-59: 2$ & -0.20 & -0.82 & $-1: 32$ & +0.03 \\
\hline 2 & 26 & 9 & 76.0 & -40.9 & -3.92 & $+0.4^{2}$ & -0.78 & +0.50 \\
\hline 3 & 31 & 4 & 187.1 & -52.3 & +0.80 & +0.08 & -0.22 & -0.28 \\
\hline 4 & $3^{1}$ & 6 & 31.7 & $-63 \cdot 3$ & +0.45 & -0.60 & -1.18 & -0.01 \\
\hline 5 & Jan. 3 I-Febr. 1 & 6 & 167.6 & -60.1 & +0.62 & -0.27 & -0.75 & -0.18 \\
\hline 6 & Febr. I & 5 & 184.6 & -65.3 & +1.53 & -0.03 & -0.37 & -0.35 \\
\hline 7 & Jan. $\quad 25$-Febr. 4 & 15 & 130.0 & -57.0 & - & - & - & - \\
\hline 8 & Jan. $\quad 29-$ Febr. 4 & 7 & 40.0 & -68.0 & 一 & - & - & - \\
\hline
\end{tabular}

Für die verschiedenen Meteorströme, mit Ausnahme als weniger wahrscheinlich zu betrachten sind, habe ich auch der Nrn. 7 und 8, deren Radiationspunkte 2 war aus den Be- die Elemente nach der parabolischen Hypothese berechnet obachtungen hervorgehen, wegen der langen Zeitdauer aber und, wie folgt, gefunden:

\begin{tabular}{c|c|c|r|c}
\hline Nr. & \multicolumn{1}{|c|}{$\pi$} & \multicolumn{1}{|c|}{$\delta$} & \multicolumn{1}{c|}{$i$} & \multicolumn{1}{c}{2} \\
\hline 1 & I 36.9 & $125^{\circ} .2$ & $44^{\circ} .5$ & 0.975 \\
2 & 137.9 & 126.2 & 25.3 & 0.975 \\
3 & 148.3 & 131.3 & 105.2 & 0964 \\
4 & 147.5 & 131.3 & 61.0 & 0.966 \\
5 & 149.0 & 131.3 & 84.7 & 0.962 \\
6 & 130.2 & 132.2 & 89.2 & 0.986
\end{tabular}


Von dem Beobachtungsmateriale hat bei der Bestimmung der Radianten nur etwa ein Viertel in Anwendung gebracht werden können. Überhaupt habe ich mich bemüht die Bestimmung der Position eines Radiationspunktes auf Sternschnuppen eines einzelnen Abends zu stützen. In einigen Fällen habe ich geglaubt, eine längere Dauer des Sternschnuppenschauers zu bemerken.

Von den Radiationspunkten sind wahrscheinlich nicht alle selbständige Centra für Sternschnuppentätigkeit, sondern vielmehr in Systeme verbunden. Dies könnte man besonders von den Radianten 5 und 6 behaupten.

Die Bahnen der beiden ersten Meteorströme haben einige Ähnlichkeit mit denen zweier alten, in China beob-

Helsingfors, 1902 August. achteten Kometen, wie die folgende, ohne Rücksicht auf die Verschiedenheit des Äquinoktiums gemachte, Zusammenstellung der Elemente zeigt:

$\begin{array}{lcccc} & \pi & \delta & i & q \\ \text { Meteorstrom Nr. I } & 13^{\circ} .9 & 125^{\circ} .2 & 44^{\circ} .5 & 0.975 \\ \text { Komet v. Jahre. 574 } & 143.6 & 128.3 & 46.5 & 0.963 \\ \text { Meteorstrom Nr. 2 } & 137.9 & 126.2 & 25.3 & 0.975 \\ \text { Komet v. Jahre 1092 } & 156.3 & 125.7 & 28.9 & 0.928\end{array}$

Besonders beim letzteren sind in einigen Elementen die Abweichungen beträchtlich. Sie finden jedoch znm Teil ihre Erklärung in der Unsicherheit bei der Bestimmung des Radiationspunktes, welcher aus nur einseitigen Sternschnuppen bestimmt werden mußte.

\section{Die Wiederkehr des periodischen Kometen 1896 VII (Perrine).}

H. Härdh.
Der von Perrine am 8. Dezember i 896 entdeckte Komet, welcher sich bald als elliptisch mit etwa $61 / 2$ Jahren Umlaufszeit herausstellte, wurde in seiner ersten Erscheinung bis zum 3. März 1897, also kaum drei Monate, beobachtet. Die letzte Beobachtung rührt von Wilson in Northfield (Minn.) mit dem dortigen $16 \cdot$ Zöller her und der Komet ist damals als most exceedingly faint, but certainly seen bezeichnet. An diesem Tage war $\log r=0.223, \log \Delta=0.017$. Außer meinen damaligen Bahnbestimmungen bat Herr Osten in A. N. 3478 aus 24 (von im ganzen 121 ) Beobachtungen, die sich bis zum r. März, also fast bis zur letzten Beobachtung, erstreckten, Elemente abgeleitet, welche zeigten,

$$
\begin{aligned}
& \text { Jupiterstörungen } \\
& \text { I } 897 \text { Febr. } 28 \\
& \text { bis } 1903 \text { Jan. } 29 \\
& +0^{\circ} \quad 9^{\prime} 32 \\
& -129.73 \\
& -4 \quad 19.41 \\
& +2 \quad 1.00 \\
& -1 \quad 1.50 \\
& -7.268 \\
& .
\end{aligned}
$$

$\left.\begin{array}{rl}L & =35^{\circ} 39: 01 \\ \pi & =5031.24 \\ \delta & =24637.20 \\ i & =1340.29\end{array}\right\}$ 1900.0

Ausgangselemente der Erscheinung 1896/7 daß eine Verbesserung der mittleren Bewegung nicht sicher erlangt werden konnte. Es schien mir daher geraten, vor einer definitiven Bearbeitung der Erscheinung $1896 / 7$ abzuwarten, ob nicht aus der beobachteten Wiederkehr des

\begin{tabular}{|c|c|c|c|c|c|c|c|}
\hline 1903 & $\alpha 1903.0$ & $\delta 1903.0$ & $\log r$ & $\log \Delta$ & $\mathrm{H}$ & $\Delta \dot{x}$ & $\Delta 8$ \\
\hline Jan. 21.5 & $21^{\mathrm{h}} 23^{\mathrm{m}} \cdot 2$ & $-4^{\circ} 33^{\prime}$ & 0.2179 & 0.4015 & 0.17 & $+I^{h} 1 I^{m}$ & $+15^{\circ} \cdot 4$ \\
\hline 29.5 & 2 I $44 . \mathrm{I}$ & -35 & 0.2010 & 0.3929 & 0.20 & +o 59 & +15.0 \\
\hline Febr. 6.5 & $22 \quad 5.9$ & -127 & o. I 840 & $0.3^{8} 56$ & 0.22 & +048 & +14.3 \\
\hline 14.5 & $22 \quad 28.0$ & $+02 I$ & 0.1670 & 0.3780 & 0.25 & +o 39 & +13.7 \\
\hline 22.5 & $22 \cdot 5^{2 \cdot 5}$ & +217 & 0.1501 & 0.3703 & 0.28 & $+0.3^{2}$ & + I 2.6 \\
\hline März 2.5 & $23 \times 7.4$ & +420 & o.1 337 & $0.3^{627}$ & $0.3 \mathrm{I}$ & +027 & +11.7 \\
\hline April 3.5 & 19.0 & +1247 & 0.0806 & $0.33^{8} 5$ & 0.44 & to 22 & +7.7 \\
\hline Mai & $\begin{array}{ll}3 & 12.7\end{array}$ & +1849 & 0.0690 & 0.3354 & 0.47 & to 25 & +2.7 \\
\hline Juni & $5 \quad 25.0$ & +1927 & 0.1070 & 0.3576 & $0.3^{6}$ & to 30 & $-3 \cdot 1$ \\
\hline Juli & $7 \quad 15.3$ & +1517 & 0.1710 & $0.395^{2}$ & 0.23 & to 8 & -7.2 \\
\hline Aug. $\quad 9.5$ & 843.2 & +848 & $0.23^{82}$ & 0.4342 & 0.14 & $-03^{I}$ & -7.2 \\
\hline Sept. I0.5 & $9 \quad 52.7$ & + I 44 & 0.2994 & $0.4^{6} 35$ & 0.09 & - I 20 & -3.4 \\
\hline Okt. I 2.5 & 10 48.3 & -54 & 0.3528 & 0.4770 & 0.07 & -220 & +2.2 \\
\hline Nov. $\times 3.5$ & I I 31.5 & 一 IIII & $0.399 \mathrm{I}$ & 0.4720 & 0.06 & -340 & +6.6 \\
\hline
\end{tabular}
Kometen sich vorweg ein sicherer Wert von $\mu$ ableiten lasse. Ich habe daher mit den Elementen des Herrn Osten die Störungen durch Jupiter von 1897 Febr. 28 bis 1903 Jan. 29 berechnet und Herr Osten hat aus freien Stücken dieselben Störungen unabhängig kontrolliert, wofür ihm mein lebhafter Dank gebührt. Damit ergaben sich folgende oskulierenden Elemente des Kometen für die diesjährige Ercheinung:

Damit ergibt sich folgende Aufsuchungsephemeride:
Oskulierende Elemente

für I 903 Jan. 29.0

auf $1903.0 \quad$ Perihel 1903 April 26.6

$\left.\begin{array}{rrr}+2.51 & 35^{\circ} 50.84 \\ +2.51 & 49 & 4.02 \\ +2.61 & 242 & 20.40 \\ -0.01 & 15 & 41.28\end{array}\right\}$ I 1903.0

$543 " 733$

0.54313 